\section{NOVA TELLVS}

\section{whts:}

Nova Tellus

ISSN: 0185-3058

novatelu@servidor.unam.mx

Centro de Estudios Clásicos

México

GONZÁLEZ VARELA, José Edgar

Los argumentos a partir de las ciencias en el Peri ideon

Nova Tellus, vol. 28, núm. 2, 2010, pp. 103-144

Centro de Estudios Clásicos

Distrito Federal, México

Disponible en: http://www.redalyc.org/articulo.oa?id=59120919005

- Cómo citar el artículo

- Número completo

- Más información del artículo

- Página de la revista en redalyc.org

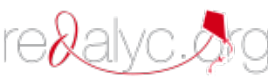

Sistema de Información Científica

Red de Revistas Científicas de América Latina, el Caribe, España y Portugal

Proyecto académico sin fines de lucro, desarrollado bajo la iniciativa de acceso abierto 


\title{
Los argumentos a partir de las ciencias en el Peri ideōn
}

\author{
José Edgar GonZÁLEZ VARELA \\ Universidad Nacional Autónoma de México \\ joedgova@gmail.com
}

\begin{abstract}
RESUMEN: En el tratado Sobre las Ideas (Peri ideōn) Aristóteles discute críticamente cinco argumentos platónicos a favor de la existencia de Ideas. En Metafísica A, 9 el mismo autor indica que tres de éstos son menos rigurosos que los otros dos. La interpretación usual sobre el Peri ideōn sostiene que su menor rigor se debe a que no son válidos para postular Ideas. En este trabajo propongo, contra esa tesis, una nueva interpretación del primer grupo de argumentos "menos rigurosos": los argumentos a partir de las ciencias. De acuerdo con nuestra interpretación éstos son, en conjunto, válidos para concluir la existencia de Ideas.
\end{abstract}

\section{The Arguments from the Sciences in the Peri ideōn}

ABSTRACT: In the treatise On Ideas (Peri ideōn) Aristotle critically discusses five platonic arguments in favour of the existence of Ideas. In Metaphysics A, 9 the same author indicates that three of these arguments are less accurate than the other two. The usual interpretation of the Peri ideōn holds that this is due to the fact that they are not valid to establish Ideas. Against this claim, in this paper I propose a new interpretation about the first group of "less accurate" arguments, the arguments from the sciences. According to my proposal these are, jointly, valid to conclude the existence of Ideas.

Palabras ClaVe: Aristóteles, ciencia, conocimiento, Ideas, Platón.

KEYWORDS: Aristotle, science, knowledge, Ideas, Plato.

RECEPCIÓN: 27 de julio de 2010.

ACEPTACIÓN: 30 de septiembre de 2010. 


\title{
Los argumentos a partir de las ciencias en el Peri ideōn
}

\author{
José Edgar GonZÁlez VARELA
}

\section{Introducción}

El tratado Sobre las Ideas (Peri ideōn) era una obra de Aristóteles dedicada a discutir críticamente la teoría platónica de las Ideas. Este tratado no nos ha llegado de manera directa. Lo conocemos a través del comentario de Alejandro de Afrodisia (ss. II-III d. C.) a la Metafísica. Alejandro cita, o al menos parafrasea, partes del tratado para expandir algunas escuetas alusiones en Metafísica A, 9. La parte más importante, filosóficamente, del Peri ideōn es la discusión de cinco argumentos platónicos a favor de las Ideas. Estos argumentos son: (i) los argumentos a partir de las ciencias, (ii) el argumento de lo uno sobre muchos, (iii) el argumento del objeto del pensamiento, (iv) el argumento de los relativos y (v) el argumento que lleva al tercer hombre. Sobre éstos Aristóteles afirma en Metafísica A, 9, pero no en lo que conservamos del Peri ideōn, que (iv) y (v) son "más rigurosos" que (i-iii). Sin embargo, las razones de esta división no son claras. En Metafísica A, 9 Aristóteles sólo afirma que:

de los modos en que mostramos que existen las Formas, ninguno de éstos parece mostrarlo. A partir de unos no se genera con necesidad una conclusión, y a partir de otros se generan Formas, incluso de las cosas que no pensamos que haya Formas. ${ }^{1}$

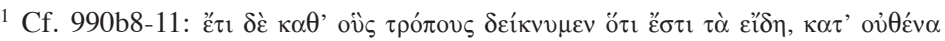

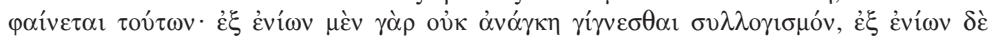


Pero no es evidente cómo deben interpretarse estas dos objeciones, ni si éstas se aplican, la primera sólo a los argumentos menos rigurosos y la segunda a los más rigurosos. ${ }^{2}$

La interpretación más influyente sobre esta distinción sostiene que Aristóteles piensa, correctamente, que los argumentos menos rigurosos: (a) no son válidos para postular Ideas, sino que, (b) sólo son válidos para postular universales aristotélicos, mientras que, (c) los argumentos más rigurosos son válidos para postular Ideas pero no son sólidos. ${ }^{3}$ En este trabajo me ocupo de estudiar el primer grupo de argumentos "menos rigurosos", los argumentos a partir de las ciencias. Aristóteles dirige en el Peri ideōn dos críticas a estos argumentos. La primera parece atacar la validez de los mismos: "tales argumentos no muestran lo que se proponen, es decir, que las Ideas existen, sino que muestran que existen ciertas cosas aparte de las cosas particulares y sensibles". ${ }^{4}$ Mientras que la segunda parece cuestionar su potencial solidez, pues Aristóteles parece detectar una inconsistencia entre una consecuencia de estos argumentos y otra tesis platónica. Según Aristóteles, si estos argumentos fueran válidos para postular Ideas, de ellos se seguiría la existencia de Ideas de artefactos, pero los platónicos rechazan tales Ideas. Así, estas críticas aristotélicas parecen corroborar la interpretación tradicional sobre la distinción rigurosa en el caso de estos argumentos.

No obstante, me parece que esta explicación no se aplica de manera tan fácil a los argumentos de las ciencias. La tesis

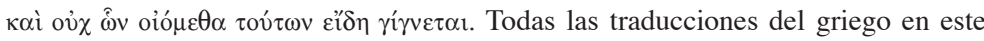
trabajo son mías.

${ }^{2}$ Entre otras cosas porque Aristóteles aplica en el Peri ideōn, de algún modo, la segunda objeción a todos los argumentos: todos darían lugar a más Ideas de las que es conveniente postular.

${ }^{3}$ Ésta parece ser la opinión de Alejandro, véase Alexander of Afrodisias... I, 78.1-25, en Dooley (1989). También defienden esta interpretación Fine (1993, pp. 25-27) y, al parecer, Santa Cruz, Crespo y Di Camillo (2000), pp. 24, 26, 28, 30 y $32-33$.

$479.16-7$. 
principal que defiendo en este trabajo es que estos argumentos son, en conjunto, válidos para postular la existencia de Ideas. Si esto es correcto, entonces la primera objeción de Aristóteles es injusta. Sin embargo, es entendible que Aristóteles no reconozca la validez de los argumentos, pues ésta depende de una premisa implícita y, paradójicamente, de una premisa extraída de la segunda crítica de Aristóteles.

En la siguiente sección, (2), presento el texto griego y mi traducción de los argumentos a partir de las ciencias y de las críticas de Aristóteles. Después, en (3), analizo los tres argumentos en conjunto. En (4) discuto, y descarto, una posible vía para establecer la validez de los argumentos. Finalmente, en (5) examino la segunda crítica aristotélica, la cual es clave para apreciar que estos argumentos son, en conjunto, válidos para afirmar la existencia de Ideas. Allí mismo discuto también si los argumentos son, además, sólidos.

\section{Texto y traducción ${ }^{5}$}

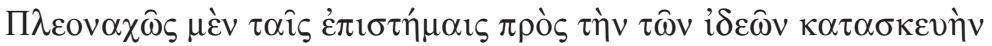

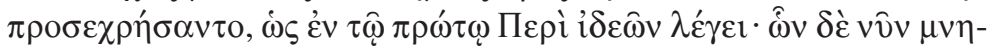

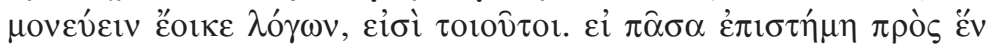

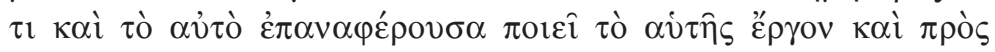

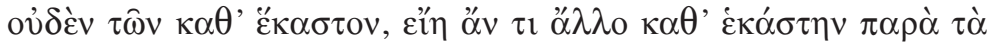

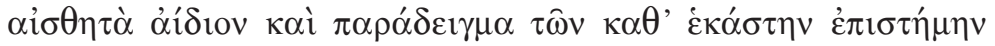

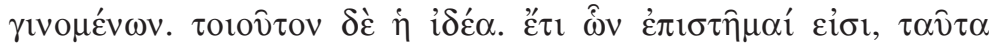

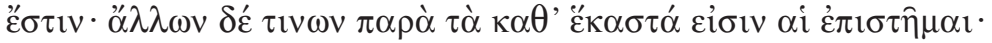

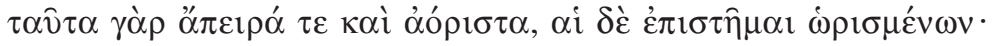

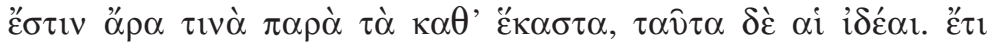

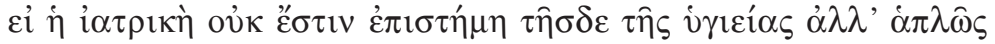

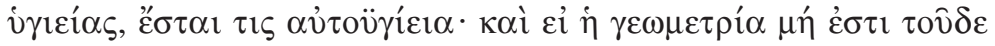

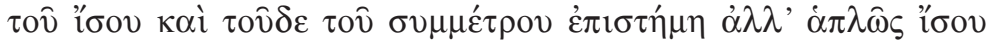

\footnotetext{
${ }^{5}$ El pasaje del Peri ideōn citado y traducido en este trabajo es el que va de 79.3 a 80.7, de Fine (1993), quien a su vez reproduce el de Harlfinger (1975).
} 


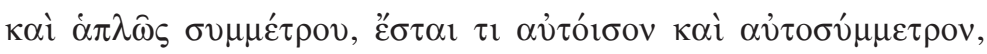
$\tau \alpha \hat{\tau} \tau \alpha \delta \dot{\varepsilon} \alpha i$ i $\delta \dot{\varepsilon} \alpha$.

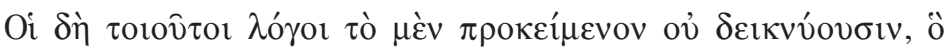

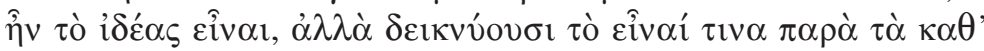

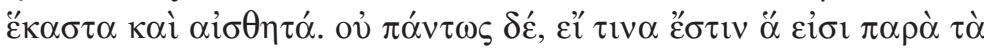

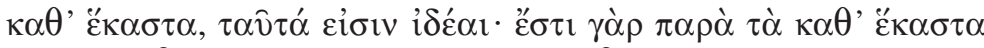

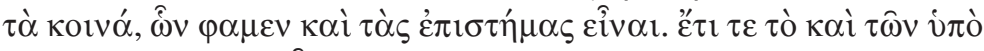

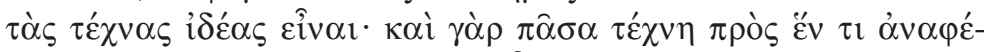

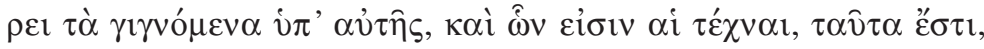

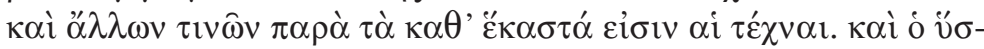

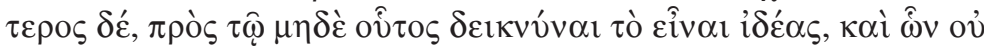

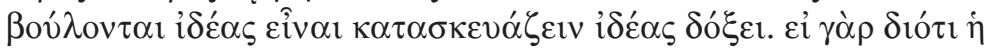

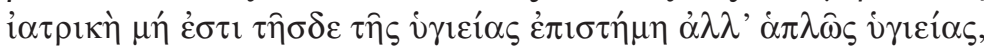

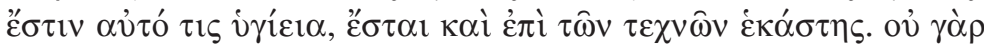

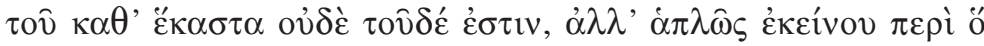

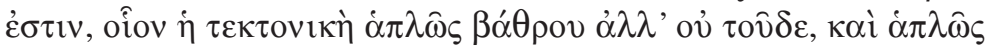

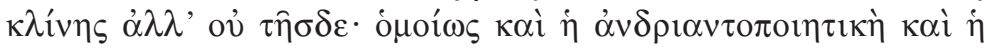

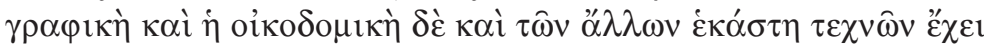

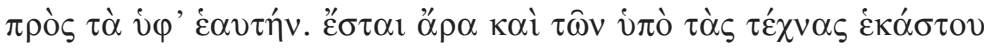

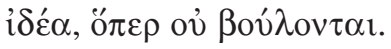

De varias maneras usaron las ciencias para el establecimiento de las Ideas, como dice en el libro primero de Sobre las Ideas, y los argumentos que parece recordar ahora son tales:

$<$ Argumentos $>$

$<1>$ Si toda ciencia realiza su función propia refiriéndose a algo uno y lo mismo, y no a ninguna de las cosas particulares, habrá según cada ciencia alguna otra cosa aparte de las cosas sensibles, eterna y paradigma de las cosas que llegan a ser según cada ciencia. Y tal cosa es la Idea.

$<2>$ Además, existen aquellas cosas de las cuales son las ciencias. Pero las ciencias son de algunas otras cosas aparte de las cosas particulares, pues éstas son indefinidas e indeterminadas, y las ciencias son de las cosas determinadas. 
Entonces, existen algunas cosas aparte de las cosas particulares. Y éstas son las Ideas.

$<3>$ Además, si la medicina no es ciencia de esta salud sino de la salud sin más, habrá una cierta salud en sí. Y si la geometría no es ciencia de esto igual y de esto conmensurado sino de lo igual sin más y de lo conmensurado sin más, habrá un igual en sí y un conmensurado en sí. Y éstos son las Ideas.

$<$ Críticas $>$

$<1>$ Ciertamente tales argumentos no muestran lo que se proponen, que las Ideas existen, sino que muestran que existen ciertas cosas aparte de las cosas particulares y sensibles. Pero de ninguna manera muestran que, si existen ciertas cosas las cuales son aparte $\mathrm{de}^{6}$ las cosas particulares, estas cosas son Ideas. Pues existen aparte de las cosas particulares las cosas comunes, de las cuales decimos precisamente que son las ciencias.

$<2>$ Además, mostrarían que hay Ideas de las cosas bajo el dominio de las técnicas. En efecto, también toda técnica refiere a algo uno, las cosas generadas por ella; y de las cosas que son las técnicas estas cosas existen, y de algunas otras cosas aparte de las cosas particulares son las técnicas. Y el último argumento, éste, además de que tampoco muestra que existen Ideas, parece establecer Ideas incluso de aquellas cosas que no quieren que haya Ideas. En efecto, si porque la medicina no es ciencia de esta salud sino de la salud sin más, existe una cierta salud en sí, también será así en el caso de cada una de

\footnotetext{
${ }^{6}$ Пopó puede traducirse también como "más allá de", denotando así uno de los rasgos distintivos de las Ideas, la separación. He optado por la traducción neutral "aparte de", que indica sólo distinción, pues Aristóteles acepta esta parte del argumento, ya que acepta que hay entidades $\pi \alpha \rho \alpha ́$ (distintas) de las cosas particularessensibles, pero niega que éstas sean Formas. Así, Frank (1984), nota (d), p. 23.
} 
las técnicas. Pues no son de una cosa particular o de un esto, sino de aquello sin más acerca de lo cual son. Por ejemplo, la carpintería es del banco sin más pero no de éste, y de la cama sin más pero no de ésta. De manera semejante, también la escultura, la pintura y la construcción, y cada una de las otras técnicas, están dispuestas con relación a las cosas bajo su dominio. Entonces, habrá también una Idea de cada una de las cosas bajo el dominio de las técnicas, lo cual no quieren.

\section{Preliminares}

Antes de empezar a estudiar estos argumentos sería útil decir algo sobre la distinción entre Ideas platónicas y universales aristotélicos, con el fin de apreciar más claramente los puntos básicos de diferencia entre ambos autores y, por ende, lo que está en juego en los argumentos.

Ambos, Platón y Aristóteles son realistas sobre los universales. Esto es, ellos piensan que, además de individuos particulares como Sócrates, el mundo contiene otro tipo de entidades tales como las propiedades (y quizás relaciones), por ejemplo, la justicia o la belleza. Las cuales son universales en tanto que una y la misma propiedad puede ser poseída por diversos individuos particulares. Por ejemplo, la propiedad de ser justo es algo que es compartido por Sócrates y Pericles en tanto que ambos son justos. Platón y Aristóteles piensan que estas entidades universales existen objetivamente, es decir, existen con independencia del lenguaje y del pensamiento: la existencia de los universales es un hecho, en sentido fuerte, del mundo. Así, la postura de estos filósofos es bastante cercana en aspectos fundamentales. Hay, sin embargo, algunas diferencias importantes en cuanto a los rasgos que ellos le atribuyen a los universales. Los rasgos en disputa parecen ser, fundamentalmente, dos: el paradigmatismo y la separación. Platón piensa que los universales son: (a) paradigmas perfectos y, 
(b) que existen separadamente de las cosas que los ejemplifican, mientras que Aristóteles rechaza que tengan estos rasgos.

(a) Por un lado, Platón describe a los universales, las Ideas, como paradigmas de las cosas particulares que participan de ellas, las cuales son, por ende, consideradas como imágenes de las primeras. La tesis central del paradigmatismo es que para cada predicado $F$ para el que hay una Idea, sólo la Idea de $F$ es plenamente $F$, mientras que las cosas particulares-sensibles que son $F$ nunca son plenamente $F$, sino que son $F$ en un grado inferior (a veces Platón afirma que son $F$ y no- $F){ }^{7}$ El modo preciso en que debe entenderse esta tesis del paradigmatismo de las Ideas es bastante discutido. La interpretación tradicional de ésta, la cual podría ser compartida por Aristóteles, es que Platón piensa que la Idea de $F$ es un ejemplar perfecto de ser $F$, mientras que las cosas particulares son ejemplares imperfectos de ser $F$. Por ejemplo, la tesis es que la Idea de igualdad es un ejemplar perfecto de igualdad, o que, si hay tal Idea, la Idea de humanidad es un ejemplar perfecto de humanidad. Esta tesis parece bastante cuestionable en la mayoría de los casos, pues parece más razonable proponer que los universales son sólo propiedades, pero no, al mismo tiempo, ejemplares perfectos de ellos mismos. ${ }^{8}$ Sin embargo, hay otra interpretación más reciente que propone una interpretación más plausible del paradigmatismo de las Ideas. ${ }^{9}$ De acuerdo con ésta, las Ideas son paradigmas perfectos de $F$ no en tanto que

\footnotetext{
${ }^{7}$ Véase, por ejemplo, Pl. Phd. 74a-75d y 78c-79a, $R$. V 473c11-480a13 y Smp. 210e6-211a5.

${ }^{8}$ Allen (1965) indica gráficamente lo extraño de tal interpretación del paradigmatismo: "Ahora bien, tal tesis es cuando menos singular. Los universales en sentido propio no son instanciaciones de sí mismos, perfectas o no. La Imparidad no es impar, la Justicia no es justa, la Igualdad no es igual a nada. Nadie puede acurrucarse para una siesta en la Divina Camidad; ni siquiera Dios puede acariciar a la Perridad detrás de las orejas" (p. 43). Además, como bien apunta Allen, tal interpretación parece llevar directamente a un problema serio, al regreso al infinito de las Ideas que es conocido con el nombre del "tercer hombre".

${ }^{9}$ Propuesta por Nehemas (1975 y 1979) y seguida, entre otros, por Fine (1993).
} 
son ejemplares perfectos de ser $F$, pues ellas mismas son sólo la propiedad de ser $F$. Más bien, la Idea de $F$ es plena o solamente $F$, porque es aquello que hace que las cosas que son $F$ sean $F$, pero nunca hace que las cosas que no son $F$ no sean $F$. Mientras que, por su parte, las cosas particulares-sensibles y muchas otras propiedades sensibles de menor generalidad, como devolver lo que ha sido prestado, son imperfectamente $F$, porque son $F$ y no $F$. Por ejemplo, Helena no es sólo bella, pues comparada con una diosa es fea, mientras que devolver lo que ha sido prestado es justo en algunas ocasiones, pero en algunas es injusto, como cuando se devuelve un arma a un amigo que se ha vuelto loco. ${ }^{10}$ La Idea de belleza y la Idea de justicia están, en cambio, exentas de la deficiencia correspondiente. Por ejemplo, la Idea de justicia es plenamente justa porque hace, solamente, que todas las cosas justas sean justas, pero no hace que las cosas injustas sean injustas. No pretendo aquí determinar cuál es la interpretación correcta del paradigmatismo, aunque es preciso decir que la segunda parece preferible, pues cuenta con respaldo en los diálogos platónicos y no le atribuye una tesis problemática a Platón. Más tarde volveremos a esta cuestión, pero adelanto de una vez que, a mi juicio, el paradigmatismo no es el rasgo crucial para mostrar la validez de los argumentos de las ciencias, sino la separación.

(b) Con respecto a la separación, hay también dos interpretaciones principales. Según la primera, que podríamos denominar "trascendente", las Ideas existen separadamente de las cosas particulares que las ejemplifican en tanto que no están en éstas, sino que están "más allá" de ellas en otro lugar de la realidad. La razón básica es que las Ideas existen por sí mismas, es decir, no requieren de otras cosas en las cuales tienen que estar para existir. ${ }^{11}$ La otra interpretación, que podríamos

\footnotetext{
${ }^{10}$ Cf., por ejemplo, Pl. Hp. Ma. 286a-289d y R. I 331c-d.

${ }^{11}$ Ésta es la interpretación tradicional de la separación de las Ideas. Algunos autores que ofrecen versiones refinadas y actualizadas de ésta son Morrison (1985) y Devereux (1994).
} 
denominar "modal", sostiene que la existencia separada de las Ideas consiste esencialmente en la característica modal de poder existir sin ser ejemplificada, o, de otro modo, en su independencia ontológica. Es decir, la Idea de $F$ existe separadamente de las cosas que son $F$, en tanto que es posible que exista incluso si no hay cosas que son $F .^{12}$ Evidentemente, esto es compatible con la tesis de que la Idea de $F$ esté de hecho en las cosas que son $F$, mientras es ejemplificada. Hay autores que piensan que la separación tal y como la concebían Platón y Aristóteles equivale sólo a la independencia ontológica, y que la evidencia a favor de la trascendencia o de la inmanencia es mixta en los diálogos; en algunos parece aceptarse que las Ideas son inmanentes, pero en otros, parece asumirse que son trascendentes. ${ }^{13}$ Otros autores, en cambio, sostienen que, si bien la concepción tanto platónica como aristotélica de la separación equivale a algo semejante a lo que he llamado trascendencia, las Ideas serían para Platón y para Aristóteles, también independientes ontológicamente. ${ }^{14}$ Sin entrar de lleno en esta polémica, es posible decir, de manera más o menos clara, que la independencia ontológica no implica la trascendencia, mientras que si un universal es trascendente, la afirmación de que es también independiente ontológicamente, parece ser una conclusión al menos natural, si no estrictamente implicada lógicamente. Además, Aristóteles rechaza ambas versiones de la separación de los universales; es quizás posible que piense que ambas están implicadas por la concepción platónica de la separación. Sin embargo, para los fines de este trabajo es suficiente con tomar a la separación como independencia ontológica, pues ésta es suficiente para distinguir la postura platónica de la aristotélica. ${ }^{15}$

\footnotetext{
${ }^{12}$ Fine (1984) es la principal defensora de esta interpretación.

${ }^{13}$ Así Fine (1984 y 1986).

${ }^{14}$ Así Devereux (1994).

${ }^{15}$ Aunque casi todos los autores están de acuerdo en que la independencia ontológica es un rasgo distintivo de las Ideas, es justo señalar que Frank (1984, pp.
} 
Para Aristóteles, en cambio, los universales no son, en algún sentido relevante, paradigmas perfectos, ni tampoco existen separadamente; es decir, si no hay cosas particulares que ejemplifiquen a un universal $F$, entonces ese universal no existe. ${ }^{16}$ Por estas razones, el paradigmatismo y la separación parecerían ser, en principio al menos, condiciones suficientes para afirmar que hay Ideas. Así, si un argumento consiguiera mostrar que los universales son paradigmas perfectos o que existen separadamente, sería, en apariencia, suficiente para mostrar que hay Ideas. De esta manera, en efecto, parece estar configurada la discusión de las Ideas en el Peri ideōn: los argumentos discutidos serán exitosos, si acaso consiguen mostrar que los universales son paradigmas perfectos o existen separadamente. ${ }^{17}$

\section{Los argumentos a partir de las ciencias}

\section{(4.1) Primer argumento}

(1) Toda ciencia realiza su función propia refiriéndose a algo uno y lo mismo (Sup.).

(2) Ninguna ciencia realiza su función propia refiriéndose a alguna de las cosas particulares (De 1).

(3) Existe, según cada ciencia, alguna otra cosa distinta de las cosas sensibles, la cual es (ii) eterna y (iii) paradigma de las cosas que llegan a ser según cada ciencia (De 1 y 2).

(4) Existen las Ideas (De 3).

109-117) niega que esto sea así. Para él, los universales aristotélicos son también independientes ontológicamente. Sin embargo, véase la nota siguiente.

${ }^{16}$ Esta dependencia ontológica es afirmada explícitamente, por ejemplo, en

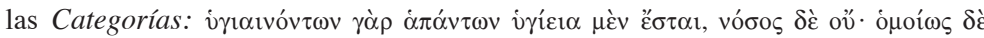

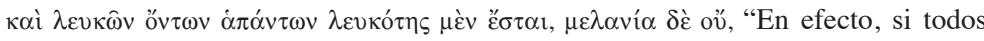
estuvieran sanos existiría la salud, pero no la enfermedad. De manera semejante también si todas las cosas fueran blancas existiría la blancura pero no la negrura" (Cat. 14a 7-10).

${ }^{17}$ Fine (1993, p. 27) defiende más ampliamente esta tesis. 
De acuerdo con (1), el desempeño de las ciencias requiere que se haga referencia a "algo uno y lo mismo". La premisa parece fijar, así, como condición necesaria para que algo sea un objeto básico de las ciencias el que éste sea uno y lo mismo. De este modo, (2) parecería excluir a las cosas particulares como objetos de las ciencias, precisamente en virtud de que éstas no satisfacen la condición fijada en (1), es decir, no son "uno y lo mismo". A partir de (1) y (2), entonces, se intenta derivar la sub-conclusión (3), y la idea parece ser que el funcionamiento de las ciencias requiere suponer que existen entidades distintas de las particulares-sensibles que son uno y lo mismo. Más adelante discutiré para qué es válido, si acaso lo es, este argumento. Por ahora es necesario ver por qué, según (1), los objetos de las ciencias necesitan ser uno y lo mismo.

La razón detrás de (1) parece ser la siguiente. Cuando una ciencia toma un conjunto de cosas que son $F$ como su objeto de estudio, la labor explicativa básica que ésta realiza requiere que se haga referencia a algo que es uno y lo mismo en todos los casos considerados, aquello que hace que todas las cosas que son $F$ sean $F$. Por ejemplo, un zoólogo que estudia reptiles requiere para determinar bien su objeto de estudio y poder explicar todos aquellos especímenes de reptiles que se le presentan, hacer referencia a algo uno y lo mismo, es decir, delimitar aquello que hace que los reptiles sean reptiles, aquello que es uno y lo mismo en todos los reptiles. Así, dado que las cosas particulares no son uno y lo mismo en este sentido, no pueden entonces ser los objetos básicos del conocimiento. Es decir, cuando una ciencia intenta explicar una serie de sucesos o hechos, la explicación no funcionaría, si se tomara como objeto básico de referencia a una cosa particular, pues ninguna de éstas puede ser aquello que explica por qué las cosas $F$ en cuestión son $F$. El argumento, no obstante, no proporciona más información sobre por qué esto no puede ser así. Sin embargo, veremos que, con mucha probabilidad, los tres argumentos no parecen sostenerse ais- 
ladamente sino que deben leerse en conjunto para ser mejor comprendidos y para evaluar de manera más justa su potencial validez y solidez. En efecto, el segundo argumento parece aportar elementos importantes para interpretar el primero y el tercero, pero éstos también son útiles para entender mejor el segundo. Así que tendremos que esperar a la discusión del segundo argumento para aclarar la cuestión mencionada. ${ }^{18}$

Ahora bien, aun teniendo este vacío explicativo, es posible adelantar un poco sobre la potencial validez de este argumento. La sub-conclusión (3) parece identificar tres puntos importantes para concluir la existencia de Ideas, la cual es inferida en (4). Por un lado, (3.i) que hay entidades distintas de las particulares y, por otro, que éstas son (3.ii) eternas y (3.iii) paradigmas. Sin embargo, parece que este argumento tan críptico y breve podría, si acaso, ser válido para concluir (3.i), pero no para concluir (3.ii) o (3.iii). Al menos es justo decir que éste no aporta elementos suficientes para realizar estas afirmaciones. Esto seguramente es así, porque el argumento no nos proporciona una explicación suficiente de por qué las cosas particulares no son los objetos básicos del conocimiento, la cual podría indicar si es que para que los universales lo sean requieren ser eternos o paradigmas. El argumento, sin embargo, parece ser válido para afirmar (3.i). Pues, por (1), las ciencias para ser posibles requieren tener cada una como objeto básico de estudio algo que es uno y lo mismo. Pero, por (2), las cosas particulares no satisfacen esta condición de ser uno y lo mismo. Así, la posibilidad de las ciencias demanda entonces que se acepte que hay entidades distintas de las particulares que puedan servir como objetos adecuados de estudio, esto es, (3.i). El argumento es, de este modo, una suerte de argumento trascendental a favor de la existencia de las Ideas, pues identifica una actividad central en nuestras

\footnotetext{
${ }^{18}$ Fine (1993, pp. 76-78) considera también que el segundo argumento es importante para entender los otros dos, pero no viceversa.
} 
vidas y después propone que ésta es posible sólo si postulamos la existencia de entidades distintas de las particulares, Ideas.

Sin embargo, según Aristóteles, aún si este argumento es válido para afirmar (3.i) no lo es para afirmar (4):

tales argumentos no muestran lo que se proponen, que las Ideas existen, sino que muestran que existen ciertas cosas aparte de las cosas particulares y sensibles. Pero de ninguna manera muestran que, si existen ciertas cosas las cuales son aparte de las cosas particulares, estas cosas son Ideas. Pues existen aparte de las cosas particulares las cosas comunes, de las cuales decimos precisamente que son las ciencias. ${ }^{19}$

La crítica de Aristóteles parece, en efecto, justa, hasta el momento y aplicable sólo a este argumento aislado, pues de (3.i), que hay entidades distintas de las particulares, no se sigue que éstas sean Ideas, pues existe la posibilidad de que sean universales que carecen de las características básicas de las Ideas, el paradigmatismo y la separación, es decir, de universales aristotélicos. Sin embargo, es preciso entender adecuadamente el sentido de la crítica de Aristóteles. La mejor manera de entender esta crítica es, contra la interpretación tradicional del Peri ideōn, considerarla como una afirmación de neutralidad. Es decir, la crítica intenta mostrar que los argumentos de las ciencias en general son válidos sólo para afirmar una conclusión neutral entre platonismo y aristotelismo: que hay entidades distintas de las particulares. Pero no sostiene que estos argumentos son válidos para afirmar la existencia de universales aristotélicos. Pues, así como no se sigue de este argumento, considerado de manera aislada, que estas entidades distintas de las particulares son Ideas, es decir, son paradigmas perfectos o existen separadamente, tampoco se sigue, por la misma razón, que no lo sean, esto es, que sean universales aristotéli-

19 79.16-19. 
cos. Más bien, el argumento no proporciona razones suficientes para afirmar una o la otra cosa y, por ende, es neutral. Y no veo ninguna razón para afirmar que Aristóteles sostenga la tesis errónea que le atribuye la interpretación tradicional, en vez de esta tesis más moderada que he propuesto.

De este modo, hemos visto que el primer argumento de las ciencias no es válido, por sí mismo, para postular Ideas. Lo cual no es muy sorprendente dada su breve extensión y carácter críptico. Sin embargo, veremos más adelante que los tres argumentos, en conjunto, parecen tener más probabilidades de ser válidos. A continuación discuto brevemente el tercer argumento.

\section{(4.2) Tercer argumento de las ciencias}

(5) La medicina no es ciencia de esta salud, sino de la salud sin más. La geometría no es ciencia de esto igual, ni de esto conmensurado, sino de lo igual sin más y de lo conmensurado sin más (Sup.).

(6) Existe una salud en sí, un igual en sí y un conmensurado en sí (De 5).

(7) La salud en sí, lo igual en sí, y lo conmensurado en sí, son Ideas (Sup.).

(8) Las Ideas existen (De 7).

Según (5), la medicina no tiene como objeto de estudio "esta"

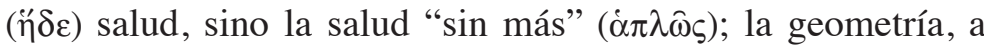
su vez, no tiene por objeto de estudio "esto" igual ni "esto" conmensurado, sino lo igual "sin más" y lo conmensurado "sin más". A partir de esta premisa se pretende llegar a la subconclusión (6): existe la salud "en sí" ( $\alpha$ vóó), lo igual "en sî", y lo conmensurado "en sî". Entidades que el argumento identifica con las Ideas. Este argumento a partir de las ciencias es diferente a los otros dos en algunos aspectos. En primer lugar, es el único que proporciona ejemplos concretos de ciencias: la 
medicina y la geometría. Además, este argumento no contiene premisas generales sobre el funcionamiento de las ciencias, sino que se basa en los casos concretos de ciencias mencionados para establecer (6). Sin embargo, parece que es razonable suponer que está implícito que estas consideraciones son aplicables al caso de las demás ciencias.

La contraposición entre lo que es "esto" ( $\tau$ ó $\delta \varepsilon)$ y lo que es "sin más", "simplemente" o "absolutamente" ( $\dot{\alpha} \pi \lambda \hat{\omega} \varsigma)$ puede significar tanto en Platón como en Aristóteles dos cosas (a veces ambos suelen utilizar $\tau \imath$ en vez de $\tau o ́ \delta \varepsilon)$. Por un lado, marca la distinción entre lo particular y lo universal, por ejemplo, distingue entre un hombre particular, Sócrates, que es "este" hombre, y el universal humanidad, que es hombre sin más. ${ }^{20}$ Por otro lado, distingue entre universales de mayor y menor generalidad, por ejemplo, entre la virtud, que es virtud sin más, y la valentía que es esta virtud o una virtud. ${ }^{21}$ Este breve argumento no proporciona indicios sobre cuál de los sentidos es relevante. Así, este tercer argumento, al igual que el primero, identifica a lo universal (o al menos a lo más universal) con aquello que es el objeto de las ciencias, pero tampoco nos dice por qué esto es así, por qué sólo aquello que es $F$ sin más o simplemente $F$, pero no aquello que es este $F$, es objeto básico de las ciencias. Sin embargo, veremos que, de igual manera que en el caso del primer argumento, el segundo argumento nos ayudará a llenar este vacío explicativo.

Con respecto a la validez del argumento, es importante apreciar que éste no concluye explícitamente la existencia de entidades distintas de las particulares como paso previo para concluir que hay Ideas. Lo que el argumento concluye es que existe la salud en sí ( $\alpha$ vó), lo igual en sí, etcétera. El término $\alpha$ vó puede ser usado por Aristóteles para designar, por un

${ }^{20}$ Véase, por ejemplo, Arist., Cat. 1b 3-9, Cael. 278a13-14, GC 317b5-7; Pl., R. 597a1-d3.

${ }^{21}$ Así, por ejemplo, Arist., Top. 123b34-35; Pl., Men. 73. 
lado, de manera neutral a lo universal por oposición a lo particular y, por otro, para designar el rasgo distintivo de las Ideas, la separación, y por tanto a estas entidades. ${ }^{22}$ Quizás los platónicos entendían el argumento de acuerdo con este segundo sentido. Sin embargo, Aristóteles debe entender av̉ có sólo en el primer sentido, pues recordemos que afirma que este argumento no demuestra la existencia de Ideas, pero sí demuestra la existencia de entidades distintas a las particulares. Es decir, Aristóteles acepta (6), que existe la salud en sí, etcétera, pero rechaza (7-8), que éstos sean Ideas y que existan, por tanto, las Ideas. ${ }^{23}$

Parece claro que, si este argumento es, por sí mismo, acaso válido, lo será sólo para afirmar una conclusión neutral entre platonismo y aristotelismo: que existen entidades distintas de las particulares. Pues, de acuerdo con el argumento, sólo aquello que es $F$ sin más o simplemente $F$ es objeto básico de las ciencias. Pero, el argumento parece suponer que las cosas sensibles no son $F$ sin más, pues son este $F$. Así, el argumento parece proponer que es necesario que existan entidades que son $F$ sin más y éstas sólo pueden ser entidades distintas a las particulares. Pero, como bien apunta Aristóteles, de esta conclusión no se sigue que existan Ideas; es decir, el argumento no proporciona elementos suficientes para determinar si, para cumplir su rol epistemológico, los universales deben ser separados o paradigmas, o si por el contrario deben ser noseparados o no-paradigmas. Tendremos que esperar para ver si el segundo argumento proporciona más indicios para decidir si los tres argumentos en conjunto, si no aisladamente, son válidos para concluir algo más que la conclusión neutral que he señalado.

\footnotetext{
${ }^{22}$ Así Fine (1993, p. 78). Por otro lado, Vlastos (1987) defiende la tesis de que Platón utiliza técnicamente el término av̉ó para indicar la separación de las Ideas.

${ }^{23}$ Fine (1993, ibid.) defiende esta tesis.
} 


\section{(4.3) Segundo argumento}

(9) Los objetos de las ciencias existen (Sup.).

(10) Las cosas particulares son indefinidas e indeterminadas (Sup.).

(11) Los objetos de las ciencias son determinados (Sup.).

(12) Existen entidades distintas de las cosas particulares (De 9-11).

(13) Las Ideas existen (De 12).

Este segundo argumento a partir de las ciencias es más explícito y riguroso que los otros dos. La premisa (9) enuncia un supuesto implícito en los otros dos argumentos, que, dado que hay ciencias éstas tienen un objeto de conocimiento, y este objeto de hecho existe. Es decir, se asume que el conocimiento es posible y que, por lo tanto, debe tener objetos sobre los cuales versa, los cuales, como afirma (11), ${ }^{24}$ deben tener una naturaleza adecuada, deben ser "determinados". Pero, según (10) las cosas particulares no poseen este rasgo necesario para ser un objeto de conocimiento, pues son indefinidas e indeterminadas. Pero como, por (9), existen entidades que son objetos de las ciencias, estas entidades deben ser, entonces, distintas de las particulares. Por lo tanto, se sigue válidamente (12), que existen entidades distintas de las particulares, esto es, universales. El argumento concluye entonces (13), que existen las Ideas.

El argumento parece ser, así, válido para afirmar (12). Pero, como hemos visto, una conclusión del tipo de (12), semejante a la conclusión (3.i) del primer argumento, es, como sostiene Aristóteles, insuficiente para mostrar que hay Ideas, pues parece más bien neutral entre su propuesta y la de Platón. Así, la inferencia de (12) a (13) parecería inválida. Más adelante examinaré si esto es correcto. Antes, no obstante, me ocuparé de las premisas (9-11).

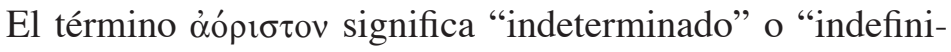
do", y puede tener un sentido cuantitativo o uno cualitativo.

\footnotetext{
${ }^{24}$ Cf. Arist. R. V 476e-477a.
} 
De acuerdo con el sentido cuantitativo, lo que es indeterminado puede ser el número de una clase en particular, por ejemplo, el número de invitados a un banquete. Según el sentido cualitativo, lo que es indeterminado sería la naturaleza misma del objeto, el modo de poseer sus propiedades, su modo de

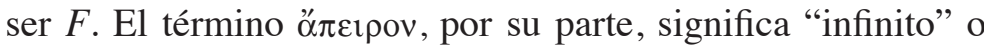

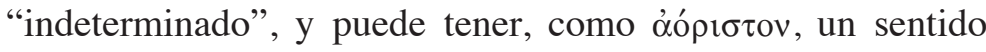
cuantitativo y uno cualitativo. Aparentemente, estos términos, aunque tienen matices un tanto diferentes, $\ddot{\alpha} \pi \varepsilon ı \rho v$ tiene más

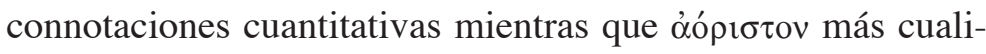
tativas, son tomados como equivalentes en el argumento, pues cuando se contrasta a los objetos de las ciencias que están exentos de estos dos rangos, se les describe solamente como

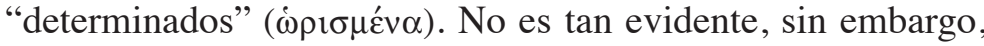
cuál es el sentido relevante y cómo debe interpretarse éste.

Pienso que el sentido cualitativo es el que está en juego en el argumento, pues, además de que esta interpretación tiene apoyo en las obras de Platón y Aristóteles (en pasajes que citaré más adelante), el sentido cuantitativo haría de éste un mal argumento. En efecto, el hecho de que el número de las cosas sensibles sea infinito o indeterminado no es por sí mismo problemático, ni es tampoco una razón para negar que estas entidades sean cognoscibles. Si bien cuando un conjunto dado de entidades tiene una cardinalidad infinita es imposible conocer todas y cada una de estas entidades, tal infinitud numérica no podría ser un obstáculo para afirmar que cada una de éstas es, en principio, cognoscible.

Así, la tesis que el argumento afirma es que los objetos de las ciencias deben ser determinados cualitativamente. ¿Por qué esto es así? Es posible responder a esta pregunta conectando (10-11) con premisas claves del primer y el tercer argumento de las ciencias y con un pasaje de la Retórica que Fine (1993) ha identificado como relevante para este argumento. El pasaje de la Retórica de hecho parece conectar explícitamente la premisa (5) del tercer argumento con la premisa (11) del 
segundo, lo cual muestra que para Aristóteles, al menos, hay una conexión natural entre ambas. El pasaje de Retórica es el siguiente:

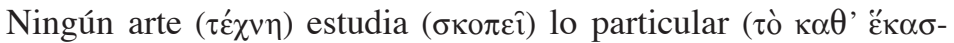
$\tau o v)$, por ejemplo, la medicina no estudia qué es lo saludable para Sócrates o para Calias, sino qué lo es para alguien de tal clase o para algunos de tales clases (pues esto es lo propio del arte, pero lo particular es indeterminado ( $\alpha$ $\pi \varepsilon i \rho o v)$ y no es cognoscible

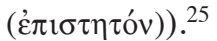

La explicación de esta conexión entre (5), (11) y el pasaje de la Retórica parece ser la siguiente. La medicina no tiene como objeto de estudio a Sócrates y su salud particular, qué puede hacer saludable a Sócrates, sino la salud en general, la salud sin más, o una clase de salud, por ejemplo, la salud del corazón. La razón es que la ciencia requiere de una cierta generalidad. La cardiología no podría, por ejemplo, realizar una explicación satisfactoria en términos sólo de la salud del corazón de Sócrates, sino, de manera primordial, en términos de la salud del corazón sin más. Es decir, su interés básico radica en explicar qué es lo saludable para el corazón humano en general, sin más, y sólo derivadamente en qué es lo saludable para el corazón de Sócrates. Esta generalidad es requerida para que la cardiología tenga un poder explicativo amplio, lo cual no podría conseguirse estudiando sólo un corazón en particular. La indeterminación como causa de la inadecuación de lo particular como objeto de conocimiento parece consistir, entonces, en que lo particular es indeterminado en tanto que contiene rasgos irrelevantes para la explicación en cuestión. Por ejemplo, un corazón en particular, digamos el de Sócrates, y su salud no son el objeto (básico) de la cardiología, porque

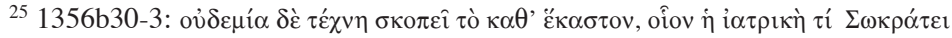

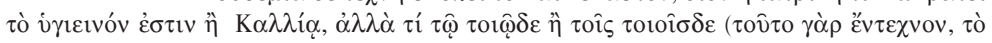

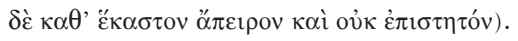


contienen rasgos que son irrelevantes para explicar la salud del corazón humano sin más, rasgos peculiares a Sócrates, rasgos que no tienen relación con la salud del corazón humano en sí. Quizás es útil recordar, en este contexto, otra de las

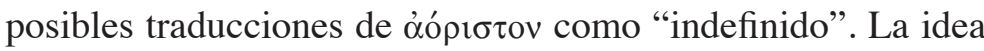
sería entonces que la medicina, al buscar una definición de la salud del corazón, no lo hace en términos del corazón particular de Sócrates, porque éste es "indefinido", es decir, tiene rasgos que no pueden caber dentro de la definición de la salud del corazón en general.

La premisa (1) del primer argumento de las ciencias es relevante también, pues es posible apreciar ahora por qué los objetos de la ciencia requieren ser uno y lo mismo. En la explicación que realiza la medicina de la salud del corazón su objeto fundamental es uno y lo mismo, el corazón sin más, pues es ésta referencia a una y la misma cosa la que hace que pueda aplicarse de manera general. Si su objeto fueran el corazón de Sócrates y el corazón de Platón, múltiples y diferentes, no tendría tal poder explicativo, pues éstos tienen rasgos que son irrelevantes para la explicación del corazón sin más. $^{26}$

Así, vemos que parece haber una conexión importante entre las premisas de los tres argumentos a partir de las ciencias. Esto justifica, a mi juicio, la pregunta de si los tres argumentos, en conjunto, podrían ser válidos para postular Ideas.

\footnotetext{
${ }^{26}$ Es importante advertir que la explicación ofrecida en este trabajo de por qué las Ideas son los objetos del conocimiento deja abierta la posibilidad de que las cosas particulares-sensibles sean, en algún sentido, cognoscibles. Si bien según estos argumentos, estas cosas son por sí mismas y directamente, incognoscibles para Platón, estas cosas son, sin embargo, indirectamente cognoscibles: una cosa sensible podría ser conocida a través del conocimiento de las Ideas que ésta ejemplifica. Por ejemplo, el conocimiento de la Idea de salud podría proporcionar los medios para conocer algo sobre Sócrates, por ejemplo, si su corazón está sano o no. Cf. Fine (1993, pp. 67, 76, 78).
} 
NOVA TELLVS, 28+2, 2010, pp. 103-144

\section{Determinación, paradigmatismo y eternidad}

Con el objetivo de examinar si los tres argumentos, en conjunto, son válidos para la postulación de Ideas formulo a continuación una versión unitaria de argumento a partir de las ciencias que puede extraerse fácilmente de los tres argumentos platónicos.

(14) Los objetos de las ciencias existen (Sup.).

(15) Los objetos de las ciencias son uno y lo mismo, $F$ sin más y no este $F$, y determinadamente $F$ (Sup.).

(16) Las cosas particulares no son uno y lo mismo, no son $F \sin$ más sino este $F$, y son indeterminadamente $F$ (Sup.).

(17) (i) Existen entidades distintas de las particulares que son (ii) eternas y (iii) paradigmas (De 14-16).

(18) Existen las Ideas (De 17).

La cuestión que me ocuparé de discutir es si acaso las premisas (14-16) son suficientes para afirmar la sub-conclusión (17. ii) y/o la sub-conclusión (17.iii), pues ya vimos que los tres argumentos son, incluso por separado, suficientes para afirmar (17.i). Después discutiré si alguna de estas sub-conclusiones es suficiente para afirmar la existencia de Ideas.

\section{(5.1) Paradigmatismo}

Como hemos visto, la premisa (15) afirma una condición que todo objeto de conocimiento debe poseer, y la cual, de acuerdo con (16) sólo puede ser satisfecha por entidades distintas de las particulares, universales, los cuales, por (14), deben existir. Así pues, la conclusión es que existen universales que son uno y lo mismo, $F$ sin más y determinadamente $F$. Como vimos en la sección anterior, esta triple condición parece consistir, al menos en parte, en no poseer rasgos irrelevantes para la explicación de qué es lo $F$. Esta condición triple que, para abreviar, denomi- 
naré "condición de la determinación cualitativa", parece estar ligada directamente con las tesis de la imperfección del mundo sensible y la de la denominada "co-presencia de opuestos".

La tesis de la co-presencia de opuestos que afecta a las cosas particulares es una premisa que figura de manera importante en algunos de los principales pasajes de los diálogos platónicos en donde se pretende argumentar a favor de la existencia de Ideas. ${ }^{27}$ De acuerdo con éstos, las cosas particulares están afectadas de una cierta deficiencia; ésta consiste en que una cosa sensible que es $F$ nunca es solamente $F$, sino que también es no$F$, al mismo tiempo. Por ejemplo, una mujer bella, Helena, no es solamente bella, sino que es también fea, o no-bella, al mismo tiempo: bella comparada con las demás mujeres, pero fea comparada con una diosa. De manera similar, una cosa A que es igual a otra cosa B nunca es solamente igual, sino que también es, al mismo tiempo, desigual a otras muchas cosas, por ejemplo, a C. Ahora bien, de acuerdo con $R$. V, esta deficiencia impide que las cosas sensibles sean los objetos del conocimiento. La razón es que los objetos del conocimiento requieren ser plenamente $F$, es decir, estar exentos de tal co-presencia de opuestos. Pero, dado que debe haber objetos de las ciencias, se sigue que éstos deben ser distintos de las cosas sensibles. Pero estas entidades son Ideas, las cuales son las únicas que son "plenamente" $F$, nunca son $F$ y no- $F$. Por tanto, existen las Ideas..$^{28}$

Este argumento es, a primera vista, semejante a los argumentos de las ciencias. De hecho, la explicación usual de este tipo de argumentos a favor de la existencia de Formas, basados en la co-presencia de opuestos, es similar a la explicación de los argumentos de las ciencias, basados en la premisa de la in-

\footnotetext{
${ }^{27}$ Especialmente, Pl. Phd. 74a-75d y 78c-79a y $R$. V 473c11-480a13.

${ }^{28}$ Esta interpretación de $R$. V se basa en una lectura predicativa del pasaje, y es defendida convincentemente, entre otros, por Cooper (1986), Santas (1973) y White (1977 y 1984).
} 
determinación cualitativa. La razón es que, cuando buscamos explicar qué es lo $F$, la explicación no puede ser alcanzada al tener como objetos a las cosas sensibles, pues éstas son $F$ y no- $F$, es decir, tienen rasgos irrelevantes para la explicación de lo $F$, rasgos que de hecho explican por qué algo es no- $F$ y no sólo rasgos que expliquen por qué algo es $F$. Por ejemplo, si buscamos explicar qué es lo bello, si consideramos a una cosa sensible como Helena, veremos que aquello que la hace bella, que explica por qué es bella (comparada con otras mujeres), por ejemplo la forma de su nariz y sus ojos, la hace al mismo tiempo fea, explica por qué es fea (comparada con las diosas). Por el contrario, la Forma de lo $F$ es un objeto adecuado de conocimiento, porque es plenamente $F$, es decir, sólo explica por qué las cosas que son $F$ son $F$, pero nunca explica por qué las cosas que no son $F$ no son $F$, pues es aquello en lo que consiste ser $F$. Por ejemplo, la Idea de belleza es aquello en lo que consiste ser bello y, por ende, sólo explica por qué las cosas bellas son bellas, pero nunca por qué las cosas que son feas son feas.

Sin embargo, hay al menos una diferencia importante entre los argumentos a partir de las ciencias y otros pasajes platónicos donde la co-presencia, interpretada de esta manera, parece tener una función relevante. Ésta es que los pasajes de la co-presencia sólo autorizan la postulación de una cantidad limitada de Formas, a saber, Formas de propiedades relacionales (como de lo igual, lo grande) y evaluativas (como de lo bello, lo justo). Esto es así, porque, de acuerdo con esta interpretación, la co-presencia de opuestos consiste en poseer una propiedad $F$ y su contraria no- $F$ al mismo tiempo, no, además en tiempos sucesivos. Y es evidente que esta condición sólo puede ser satisfecha por propiedades relacionales y evaluativas, que de hecho figuran prominentemente en los pasajes relevantes: una misma cosa puede ser justa e injusta al mismo tiempo, igual y desigual al mismo tiempo, etcétera, siempre y cuando el aspecto en el que poseen cada una de las propieda- 
des opuestas sea diferente, o, también, el objeto con relación al cual posean cada una de las propiedades opuestas sea diferente. Por ejemplo, Helena puede ser bella (en comparación con $\mathrm{X}$ ) pero fea (en comparación con $\mathrm{Y}$ ) al mismo tiempo, sin contradicción y sin sufrir ningún tipo de cambio. Por el contrario, propiedades distintas, que, siguiendo a Aristóteles podríamos denominar "substanciales", como ser un tigre o ser una mesa, no admiten la co-presencia: no parece razonable afirmar seriamente que algo puede ser, al mismo tiempo, un tigre y no ser un tigre, ni ser una mesa y no-mesa al mismo tiempo. Pues, tales propiedades substanciales parecen estar más cercanas a la identidad de las cosas. Si $x$ es un tigre, $x$ es en esencia esto, y el llegar a ser no-tigre no representaría la posesión, por parte de $x$, de la propiedad opuesta, sino más bien la destrucción de $x$ como individuo. Por el contrario, los argumentos de las ciencias no parecen tener tal limitación, sino ser aplicables, en principio, a cualquier propiedad. De hecho, no tienen tal limitación para Aristóteles, pues, según él, éstos serían válidos para postular algunas Ideas substanciales, las Ideas de artefactos.

Sin embargo, Fine ${ }^{29}$ ha argumentado que, si bien, por las razones expuestas, la indeterminación que está a la base de los argumentos a partir de las ciencias no puede considerarse equivalente a la co-presencia, en este sentido restringido, sí puede ser equivalente a lo que podría denominarse "copresencia amplia". Tal co-presencia amplia consistiría entonces, precisamente, en que las cosas particulares son $F$ pero al mismo tiempo no son $F$, sólo en tanto que poseen rasgos irrelevantes para explicar qué es lo $F$. Es evidente que tal copresencia amplia no está limitada a propiedades relacionales y evaluativas, pues, por ejemplo, Fido, siendo un perro particular, posee rasgos irrelevantes para explicar la Perridad. Además, según Fine, tal co-presencia amplia es quizás de mayor

29 1993, pp. 100-101. 
interés para Platón que la co-presencia restringida, la cual es un caso particular de la primera, pues Platón postula Formas más allá de las que legitima la co-presencia restringida, como Ideas de cuadrado, triángulo, etcétera.

Así, la determinación que requieren los objetos del conocimiento según los argumentos de las ciencias es, para Fine, equivalente a la exención de co-presencia amplia. Pero, para Fine, estar exentos de una clase particular de esta co-presencia, de la co-presencia restringida, es precisamente aquello en lo que parece consistir el que las Ideas sean, de acuerdo con Platón, paradigmas perfectos: "nada más involucra, en realidad, el ser un paradigma perfecto que el escapar de la co-presencia". 30 Pero, entonces cabe la pregunta que Caston (1995) ha ya formulado: si los argumentos de las ciencias son, en su conjunto, válidos para establecer la existencia de universales determinados, y esta determinación es, de acuerdo con Fine y otros, precisamente en lo que consiste la perfección de las Ideas, por qué entonces éstos no son válidos para postular la existencia de universales que son paradigmas perfectos, lo cual, al parecer, es suficiente para afirmar la existencia de Ideas.

Caston (1995) sostiene precisamente esta tesis en contra de Fine (1993). Fine (1993) advierte, sin embargo, este problema que parece enfrentar su interpretación y propone la siguiente solución. De acuerdo con ella, aunque Platón probablemente habría considerado que al establecer la existencia de universales determinados, los argumentos a partir de las ciencias establecen la existencia de paradigmas perfectos y, por ende, de Ideas, Aristóteles niega que estas dos últimas afirmaciones sean válidas, porque Platón nunca formula de manera clara qué está involucrado en ser un paradigma perfecto:

Así, quizás Aristóteles no menciona la perfección en los Argumentos a partir de las Ciencias porque no siente ninguna obli-

\footnotetext{
${ }^{30}$ Fine, 1993, p. 95.
} 
gación de darle a Platón premisas que Platón asume pero no se toma el trabajo de articular claramente. Es probable que Aristóteles también crea que si el paradigmatismo perfecto no involucra nada más que lo que he sugerido, entonces, Platón debería haber descrito su punto de vista de alguna otra manera, pues sus grandiosas descripciones de las formas sugieren naturalmente una lectura más robusta. ${ }^{31}$

Fine parece sugerir aquí que no sólo no está claro, para Aristóteles, qué involucra el paradigmatismo perfecto, sino que incluso si estuviera explícito qué es lo que éste más probablemente involucra, la determinación cualitativa tal y como ha sido explicada, entonces este rasgo no sería suficiente para afirmar la existencia de universales platónicos.

Si esto es lo que sostiene Fine, estoy de acuerdo con ella. Y la razón parece obvia, la determinación cualitativa de los universales, el que el universal $F$-idad no posea rasgos irrelevantes para explicar que algo es $F$, parece ser una condición satisfecha por cualquier universal. Esta característica de hecho es parte de aquello en lo que consiste ser un universal. Si esto es así, entonces el paradigmatismo perfecto, entendido como determinación cualitativa, es insuficiente para mostrar la existencia de Ideas, porque no podría ser un rasgo exclusivo de los universales platónicos: cualquier universal lo tendría por el hecho mismo de ser un universal. Sin embargo, esta conclusión parece tener un resultado que Fine no advierte: la condición del paradigmatismo perfecto deja, entonces, de tener relevancia alguna para determinar si un argumento es válido para postular Ideas. ${ }^{32}$ Pues, si en un argumento a favor de las Ideas el paradigmatismo sólo consiste en la determinación cualitativa, éste

\footnotetext{
${ }^{31}$ Ibid.

${ }^{32}$ Fine está lejos de advertir esto, pues sigue considerando al paradigmatismo como condición suficiente para afirmar la existencia de las Ideas. Por ejemplo, de acuerdo con ella, el argumento a partir de los relativos es válido para postular Ideas porque es válido para postular paradigmas perfectos.
} 
captura correctamente el punto de vista de Platón y le atribuye una tesis plausible, pero no puede ser suficiente, por sí mismo, para afirmar la existencia de Ideas. Por el contrario, si en el argumento el paradigmatismo consiste en "una tesis más robusta", por ejemplo, que la Idea de $F$ es un ejemplar perfecto de lo $F$, entonces sí es suficiente para distinguir a los universales platónicos de otros universales, pero pagando el alto precio de darle a Platón una tesis absurda sobre la naturaleza de los universales y malinterpretando su pensamiento.

\section{(5.2) Eternidad}

Parece entonces más razonable buscar una ruta diferente a la sugerida por Caston (1995), para determinar si los argumentos a partir de las ciencias son válidos para postular Ideas. Ésta consiste en examinar si éstos son válidos para afirmar (17.ii), esto es, la eternidad de los universales.

De acuerdo con Fine (1993), la premisa de la determinación cualitativa no valida la conclusión de que los objetos de las ciencias son eternos. Esto es, el requerimiento de que los objetos de las ciencias sean determinados no incluye, propiamente, el que sean eternos, sino sólo la tesis, ya explicada de no poseer rasgos explicativos irrelevantes. Este requerimiento es, como hemos visto, para Fine, equivalente a la co-presencia amplia: los objetos de las ciencias deben ser sólo $F$, nunca $F$ y no- $F$. Incluso, según Fine, puede decirse que se requiere que estos objetos sean siempre $F$, y nunca no- $F$, en el sentido de ser $F$ en todo tiempo en el que existan, pero esto no implica que se requiera que en todo tiempo sean $F$, de donde se seguiría que existen en todo tiempo. Sin embargo, me parece que hay buenas razones para incluir a la eternidad, entendida como existencia en todo tiempo, dentro de los requerimientos involucrados en la determinación cualitativa que se requiere, según Platón, para ser objeto de conocimiento. 
En primer lugar, si la eternidad no fuera un requerimiento, esto pondría en riesgo, desde una perspectiva platónica, la estabilidad de la ciencia. Por ejemplo, supongamos que hay una ciencia $\mathrm{C}$ en un tiempo t1 que tiene por objeto al universal $F$-idad. Si no estuviera garantizada la eternidad de $F$-idad, podría ser el caso de que $F$-idad dejara de existir en t2, pero, por tanto, $\mathrm{C}$ carecería de objeto de conocimiento y dejaría de existir. Así, parece razonable pensar que los objetos de las ciencias requieren, desde el punto de vista platónico, de suficiente estabilidad. Pero, si no fueran eternos, no serían estables. En segundo lugar, la eternidad parece estar incluida, de manera natural, en la tesis misma de la determinación cualitativa, por razones ontológicas y no sólo epistemológicas. Como hemos visto, la determinación cualitativa parece ser equivalente a la perfección paradigmática y a la co-presencia amplia. Pero no parece plausible que, dentro de la perfección que Platón le otorga a las Ideas, no esté incluida la eternidad como una condición necesaria. De hecho, el contraste en términos temporales entre las Ideas que son siempre y las cosas sensibles que están sujetas al devenir es frecuente en la obra platónica. Pero hay un pasaje en esta obra que es de particular importancia, pues muestra, por un lado, que el contraste temporal entre finitud e infinitud era importante para Platón al contrastar a las Ideas con las cosas sensibles y, de manera más relevante, muestra que el ser $F$ y no- $F$, la "co-presencia" de opuestos, no involucra sólo, estrictamente, co-presencia de opuestos, sino también sucesión temporal de opuestos. En Banquete se afirma, con respecto a la Idea de lo bello que:

En primer lugar, siempre es, y no nace ni perece, ni aumenta ni disminuye; y, además, <a $>$ no es bella en un aspecto y fea en otro, ni $<b>$ bella en un tiempo y en otro no, ni $<\mathrm{c}\rangle$ bella con relación a una cosa y fea con relación a otra, $\mathrm{ni}<\mathrm{d}>$ bella aquí y fea allí, como si fuera bella para unos y fea para otros. ${ }^{33}$

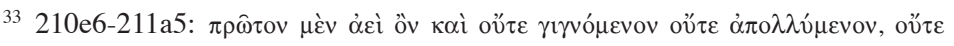


De acuerdo con este pasaje, (a), (b) y (d) pueden interpretarse como concerniendo, propiamente, a la co-presencia de opuestos, pero (2) parece enunciar la sucesión de opuestos, la cual, de acuerdo con el inicio del pasaje donde se afirma que la Idea de lo bello "siempre es y no nace ni perece", parece que debe interpretarse en el sentido fuerte de que, en todo tiempo pasado, presente y futuro, la Idea de lo bello es bella y, por ende, existe en todo tiempo. Así, parece plausible incluir a la eternidad como una condición o presuposición necesaria de la mal llamada "co-presencia" amplia, de la perfección y, por ende, de la determinación cualitativa.

Sin embargo, incluso si, como parece razonable, se debe incluir a la eternidad dentro de la determinación cualitativa, es posible argumentar que la posesión por parte de los universales de tal rasgo no es suficiente para afirmar la existencia de Ideas. Fine (1984) parece sostener en verdad esta tesis. De acuerdo con Fine, la tesis de que los universales son eternos no implica, por necesidad, la existencia de Ideas, pues, crucialmente, no implica la separación de los universales. La razón es que, según Fine, el hecho de que un universal $\mathrm{U}$ exista en todo tiempo $\mathrm{t}$ no implica que pueda existir sin que haya cosas particulares que lo ejemplifiquen, pues bien podría ser que en todo tiempo t haya cosas que ejemplifiquen a U. Así, bien podría ser que $\mathrm{U}$ requiera que haya cosas particulares que lo ejemplifiquen para existir, pero que sea, no obstante, eterno simplemente porque siempre hay cosas particulares que lo ejemplifican. De este modo, la eternidad parece no ser un rasgo distintivo de los universales platónicos, pues parece que podría ser poseído por los universales aristotélicos. De hecho, Fine ${ }^{34}$ sostiene que, para Aristóteles, los universales son, probablemente, eternos. ${ }^{35} \mathrm{Y}$,

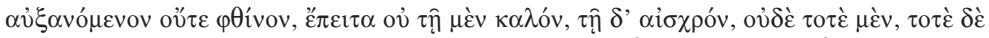

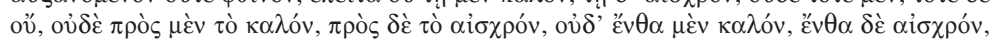

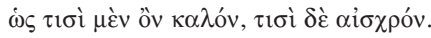

${ }^{34} 1993$, p. 283, notas 20 y 23.

${ }^{35}$ Cf. APo. 77a. 
además, que Aristóteles parece estar en condiciones de aceptar la eternidad de los universales, sin aceptar al mismo tiempo su separación, pues Aristóteles parece aceptar la eternidad del mundo material: si bien toda cosa que es $F$, para cualquier universal genuino $F$, es finita y corruptible, las cosas que son $F$, en conjunto, no lo son, pues siempre ha habido y siempre habrá una cosa que es $F$.

Creo que Fine tiene razón, al menos, en que la separación de los universales no se sigue de su eternidad tan fácilmente como podría creerse, y en que Aristóteles acepta, en efecto, que sus universales son eternos. Sin embargo, me parece que hay problemas con la premisa auxiliar que requiere la tesis de Fine, a saber, que no sólo el mundo material es, en su conjunto, eterno, sino también que, para cualquier universal genuino U, siempre ha existido y siempre existirá una cosa particular que ejemplifique a U. La idea de la extinción de las especies es, por supuesto, una tesis científicamente fundada hoy en día, $\mathrm{y}$, si bien no es una tesis empírica que los griegos asumieran, no era de ninguna manera una hipótesis inconcebible. No obstante, no voy a insistir en defender tal postura por esta vía. Mi estrategia será, más bien, mostrar que la eternidad sí implica la separación, al menos en algunos casos, y con ayuda de una premisa auxiliar que, paradójicamente, la segunda crítica de Aristóteles se encarga de establecer: que los argumentos de las ciencias son válidos para mostrar la existencia de universales de artefactos.

\section{Las Ideas de artefactos y la separación}

Hemos visto que Aristóteles dirige una segunda crítica contra los argumentos de las ciencias. Según él, si de éstos se siguiera la existencia de Ideas, también se seguiría que hay Ideas de artefactos, pero hay que considerar que ellos (Platón o los platónicos en general) no admiten este tipo de Ideas. 
Me parece que Aristóteles está en lo correcto; de estos argumentos, en efecto, se seguirían Ideas de artefactos. Sin embargo, argumentaré que esta conclusión, más la conclusión válida de que los universales son eternos, es suficiente para mostrar que los argumentos de las ciencias son válidos para postular Ideas. Por último, examinaré de qué manera la crítica, a pesar de ser, como espero mostrar, de utilidad para Platón, es, no obstante, también dañina, según Aristóteles.

\section{(6.1) Separación}

Aristóteles parece estar en lo correcto cuando afirma que los argumentos de las ciencias, si demostraran la existencia de Ideas, demostrarían la existencia de Ideas de artefactos, pues, según él, las consideraciones epistemológicas que estos argumentos aducen para postular Ideas, son aplicables también al caso de las técnicas. ${ }^{36}$ En efecto, una técnica no versa de manera especial sobre un objeto particular, sino que también aspira a cierta generalidad. La razón de fondo parece ser, de nuevo, que los objetos de las técnicas tienen que ser determinados, en el sentido de que deben carecer de rasgos explicativos irrelevantes: deben versar acerca de lo que es $F$ sin más y no acerca de un $F$ particular que posee rasgos irrelevantes para la técnica en cuestión. Por ejemplo, la carpintería debe versar acerca de la mesa sin más y no sólo acerca de "esta mesa". ${ }^{37}$ Además, aunque Aristóteles y Platón con frecuencia consideran a las técnicas como inferiores epistémicamente a las ciencias, no obstante, reconocen que son un cierto modo de conocimiento. ${ }^{38}$ Así, la crítica de Aristóteles es justa en este sentido: si los argumentos de las ciencias muestran que un universal $\mathrm{U}$ existe,

\footnotetext{
${ }^{36}$ Cf. 79.20-80.5.

${ }^{37}$ Véase $R$. X 596b y Metaph. A 981a5-b10.

${ }^{38}$ Cf. Chrm. 165d, Euthphr. 14c, Ión 537c y ss., R. I, 342c; y Hintikka (1974).
} 
entonces muestran que los universales de artefactos existen. Sin embargo, como paradoja, esta conclusión más la tesis de que los universales son eternos, es suficiente para concluir válidamente la separación de los universales y, por ende, que existen las Ideas.

Como vimos, la tesis de que los universales son eternos parece insuficiente para mostrar su separación, pues un universal $\mathrm{U}$ puede existir en todo tiempo $\mathrm{t}$, precisamente porque en todo tiempo t existen cosas particulares que lo instancian. Así, un universal puede ser eterno y no ser independiente ontológicamente. Esta tesis se apoya, según Fine, en la premisa de que el mundo es eterno y, en específico, que, para cada universal U que es eterno, los miembros de la clase que lo ejemplifican también lo son, en el sentido de que en todo tiempo existe al menos un miembro de esa clase. No obstante, aunque esta tesis parece resultar, en principio, plausible en el caso de entidades naturales como animales, plantas y minerales, la tesis no tiene ninguna plausibilidad en el caso de artefactos. En esto es claro que, incluso suponiendo la existencia en todo tiempo de seres humanos que utilizan artefactos, es poco razonable sugerir que para cada tipo de artefacto $A$, siempre ha habido y siempre habrá artefactos As. La generación y destrucción de todos los miembros de una clase de artefactos se circunscribe a un tiempo específico. Es decir, la evolución técnica es un hecho indiscutible, del que los griegos estaban al tanto (aunque no de la manera en que lo estamos hoy en día): los artefactos han sido producidos en un tiempo determinado y en el tiempo anterior a éste no existían. De la misma manera, muchas clases de artefactos que hoy existen dejarán de existir en un tiempo futuro, pues dejarán de ser producidos, debido a que su producción se vuelve ya obsoleta. Pero, entonces, si existe un universal de artefacto $\mathrm{U}$ y si éste es eterno (premisas (17.i y 17.ii), se sigue que, al menos en la gran mayoría de los casos, ha habido un tiempo t en el cual U existía y no existía ningún artefacto que lo ejemplificara. Y, en algunos casos, 
habrá en el futuro un tiempo t en el que U existirá y no habrá ningún artefacto que lo ejemplifique. Pero esto es en lo que consiste, de manera mínima, la separación de los universales para Platón y para Aristóteles, en poder existir sin que haya cosas que los ejemplifiquen. Por tanto, si, como hemos visto, los argumentos de las ciencias son, en su conjunto, válidos para postular la existencia y eternidad de los universales y para postular universales de artefactos, entonces son válidos para postular la separación de, al menos, estos universales y, por ello, para postular la existencia de Ideas.

La relación entre la postulación de Ideas de artefactos y la separación es algo que ha sido advertido con anterioridad. Por ejemplo, por Fine (1984), quien, si bien sostiene que Aristóteles considera a la separación como el rasgo distintivo de los universales platónicos, afirma que no es fácil descubrir un compromiso claro con la separación de las Ideas en los diálogos platónicos, a excepción quizás del Timeo. Sin embargo, considera que la única ruta que claramente parece comprometer a Platón con la separación de al menos algunos universales es a través de la postulación de Ideas de artefactos, por razones similares a las aquí expuestas. Sin embargo, Fine (1993) no advierte que por una ruta semejante es posible llegar a la conclusión de que los argumentos a partir de las ciencias son, en su conjunto, válidos para postular la separación y, por ende, la existencia de, al menos, algunas Ideas.

Así, hemos visto que los argumentos a partir de las ciencias son, en su conjunto, válidos para afirmar la existencia de Ideas. Sin embargo, Aristóteles sostiene que esto no es así. La razón de esta opinión es - me parece-, que Aristóteles sólo considera los argumentos por sí mismos sin tomar en cuenta el impacto que podría tener su segunda crítica para mostrar la separación de los universales, en conjunción con otras premisas plausibles implícitas en el argumento. Además, Aristóteles podría estar aplicando en este caso una política general que, según Fine (1993), sigue en el Peri ideōn, la de no concederle 
a los platónicos premisas que no están claramente formuladas ni argumentadas. Asimismo, es probable que Aristóteles no se hubiese interesado en explorar la opción de que la adición de la premisa de que del argumento se seguirían universales de artefactos (más la premisa de la eternidad) harían al argumento válido para postular Ideas, pues consideraría que, aunque así fuera, tal adición haría también al argumento inevitablemente no-sólido. Este aspecto de la segunda crítica de Aristóteles lo abordo en la siguiente sección.

\section{(6.2) Ideas de artefactos}

La segunda crítica de Aristóteles ha suscitado mucha discusión entre los estudiosos del Peri ideōn, pero con respecto a un aspecto diferente al enfatizado en la sección anterior. Esta crítica ha sido interpretada principalmente como un argumento ad hominem; el argumento mostraría que hay Ideas de artefactos, pero los platónicos no quieren que haya este tipo de Ideas: "habrá también una Idea de cada una de las cosas bajo el dominio de las técnicas, lo cual no quieren". ${ }^{39}$ Así, el aspecto principal de esta crítica no es señalar que habría un problema importante con postular Ideas de artefactos. Si bien es probable que esta tesis esté implícita en la objeción, el sentido básico de ésta, según esta interpretación, sería señalar una inconsistencia por parte de los platónicos. También se ha discutido que como objeción ad hominem la crítica parece bastante singular, pues resulta extraño que Aristóteles afirme que los platónicos no aceptan Ideas de artefactos, cuando hay dos pasajes de los diálogos platónicos en donde parece aceptarse la existencia de este tipo de Ideas. ${ }^{40}$

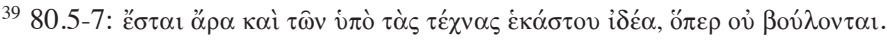

${ }^{40}$ En $R$. X 596a-598d y Cra. 389a7-8.
} 
Se han ensayado numerosas hipótesis para dar cuenta de esta objeción de Aristóteles, por ejemplo: que el Peri ideōn no discute la teoría de las Ideas de Platón, sino la de algunos platónicos que rechazaban Ideas de artefactos; ${ }^{41}$ que Aristóteles malinterpreta a Platón, pues Platón rechaza Ideas sólo de las artes imitativas (v. gr., la pintura), pero no de las artes productivas (v. gr., carpintería); ${ }^{42}$ que Aristóteles no malinterpreta a Platón, pues reconoce la distinción anterior, pero su crítica consiste en señalar que los argumentos darían lugar a Ideas de todas las técnicas, incluidas las imitativas; ${ }^{43}$ que Platón, en realidad, nunca admitió, ni deseaba hacerlo, Ideas de artefactos, ni siquiera en los pasajes donde parece hacerlo, ${ }^{44}$ etcétera. Algunas de estas hipótesis son más verosímiles que otras, y quizás podría resultar útil considerarlas, sin embargo, no pretendo entrar en esta polémica aquí, principalmente, porque creo que la objeción no es ad hominem.

Aunque Aristóteles afirma que los platónicos "no quieren (ov̉ ßov́ $\lambda o v \tau \alpha \mathrm{l}$ )" Ideas de artefactos, este verbo en modo indicativo no necesariamente describe la posición de Platón o de los platónicos. En otro pasaje del Peri ideōn Aristóteles formula una objeción en el modo indicativo, que no parece intentar describir nada sino hacer una recomendación: "este argumento establece Ideas incluso de los relativos. Al menos, la presente prueba ha sido avanzada con respecto a lo igual, lo cual es uno de los relativos; pero decían que no hay Ideas de los relativos". ${ }^{45}$ En efecto, si hay Ideas que Platón claramente admitía, éstas son Ideas de relativos, como de lo igual. Por

\footnotetext{
${ }^{41}$ Isnardi Parente (1981), quien sugiere a Jenócrates como posible candidato.

${ }^{42}$ Así, Robin (1908, p. 178), Ross (1951, pp. 173-175) y Cherniss (1944, pp. 247-260).

${ }^{43}$ Frank (1984, pp. 91-92).

${ }^{44}$ Fine (1993, pp. 85-88).

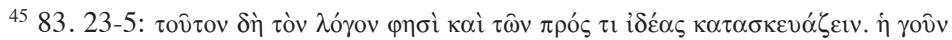

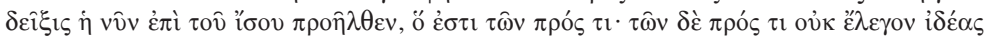
Eîvol.
} 
ello, Aristóteles no puede estar describiendo aquí la posición de Platón, sino que más bien su crítica es que los platónicos no deberían aceptar que hay Ideas de relativos. Ésta es, por ejemplo, la tesis que Fine ${ }^{46}$ defiende con respecto a esta objeción al argumento de los relativos. Me parece, entonces, que, por razones similares, la objeción a los argumentos de las ciencias debe interpretarse de la misma manera: Aristóteles no sostiene que Platón no acepta que haya Ideas de artefactos sino que no debería hacerlo. ${ }^{47}$

La razón de esta recomendación parece ser que la postulación de Ideas de artefactos contravendría nuestras intuiciones más básicas sobre la producción técnica. Al nivel del sentido común, hay una distinción importante entre objetos naturales y objetos artificiales. Al parecer, un requisito para que una teoría sea una explicación satisfactoria de la producción técnica o artística es que preserve y dé cuenta de esta distinción. Sin embargo, la postulación de Ideas de artefactos la pondría en peligro. Nuestras intuiciones nos dicen que un artefacto, a diferencia de un objeto natural, es producto del ingenio humano, de la capacidad humana de modificar su entorno natural. El ejercicio de esta capacidad está condicionado, en buena parte, por las circunstancias históricas y sociales en las que viven los seres humanos. Así, por ejemplo, la "computadora" es un invento humano que se desarrolló en un tiempo y dentro de una sociedad muy específica. Sin embargo, si se admitieran Ideas de artefactos, habría un Idea de "computadora" cuya existencia no tendría un principio ni un fin en el tiempo, y que, además, sería ontológicamente independiente de cualquier cosa particular, incluida cualquier mente humana y cualquier so-

\footnotetext{
461993 , pp. 184 y 338, n. 2.

${ }^{47}$ Fine (1993, p. 303, n. 29) contempla también la posibilidad de que Aristóteles, en su crítica al argumento de lo uno sobre muchos, al afirmar que ellos "no querían

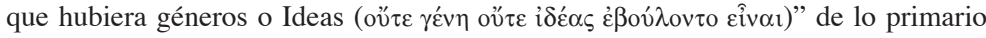
y secundario, también esté sosteniendo que no deberían aceptar tales Ideas, pues Platón sí parece aceptarlas.
} 
ciedad. Esto implicaría que los inventores de este artefacto en realidad no han inventado nada, sino que sólo han descubierto La Computadora y, por ende, los medios necesarios para producir computadoras.

Y el mismo problema se presentaría con la postulación de universales aristotélicos de artefactos, no sólo de Ideas. Pues, como he sugerido antes, los universales aristotélicos son también eternos. Por esta razón es que probablemente Aristóteles, aunque acepta que los argumentos son válidos para postular entidades distintas de las particulares, piensa que no son sólidos. Aristóteles, de hecho, proporciona en, otras obras, razones para negar que existan universales objetivos de artefactos, las cuales parecen buscar preservar las intuiciones básicas mencionadas sobre la distinción entre lo natural y lo artificial. En Física II, 1 y en Metafísica Z, 7-9, Aristóteles elabora una distinción entre objetos naturales y objetos artificiales, según la cual no hay universales objetivos de artefactos. Esta distinción consiste en que, en el caso de los objetos naturales, su forma es "interna" en el sentido de que es una forma que ninguna mente humana puso ahí y que se transmite, en muchos casos, de un miembro de la misma especie a otro. ${ }^{48}$ De este modo, la forma de un objeto natural es un universal objetivo, independiente de la mente y del lenguaje. Sin embargo, en el caso de los objetos artificiales, Aristóteles afirma que su forma no es más que el concepto en la mente del artesano y en este sentido es "externa" al objeto artificial o puesta en él desde afuera (y no transmisible de un objeto artificial a otro). ${ }^{49}$ Así, por ejemplo, una cama de madera ejemplifica muchos universales objetivos, por ejemplo, las propiedades concernientes a su madera, su figura, su color, pero, de ninguna manera ejemplifica el universal objetivo cama, pues no hay tal universal. Lo que ocurre, según Aristóteles, es que alguien concibe la

${ }^{48}$ Ph. II.1. 192b12-33, 193b2-7, Metaph. Z. 7. 1032a15-25.

${ }^{49}$ Ph. II.1. 193b2-12, Metaph. Z. 7. 1032b1-30. 
noción de una cama y después modifica ciertos objetos naturales de acuerdo con esta noción, pero esta noción nunca deja de ser una noción, jamás se vuelve un universal en los objetos extra-mentales.

Éstos son, pues, los problemas a los que parece dar lugar la postulación de Ideas (y en general universales objetivos) de artefactos, y la manera como Aristóteles evitó la postulación de éstos. Tal parece entonces que, si bien la adición de la premisa de que los argumentos de las ciencias son válidos para postular universales de artefactos haría a éstos válidos para postular Ideas, esta misma adición los condenaría a ser no-sólidos. Sin embargo, Platón y otros platónicos parecen haber estado en algún momento dispuestos a vivir con el costo que implicaría la postulación de estas Ideas, a saber, el rechazo de una tesis aparentemente básica sobre la distinción entre lo natural y lo artificial. La disputa última, entonces, entre Platón y Aristóteles sobre los argumentos a partir de las ciencias debería ser sobre la posibilidad de aceptar este costo teórico en el que los argumentos incurrirían.

\section{Conclusión}

Hemos visto que, en contra de las interpretaciones usuales de los argumentos de las ciencias, éstos son válidos, en conjunto, para postular Ideas y no sólo para una conclusión neutral entre platonismo y aristotelismo (y mucho menos son sólo válidos para postular universales aristotélicos). La clave para mostrar esto radica no en la tesis del paradigmatismo de las Ideas - como piensa Caston (1995) - , sino en la de la separación. Paradójicamente, la segunda crítica de Aristóteles, que los argumentos serían válidos para postular universales de artefactos, es la pieza básica para mostrar que los argumentos son, en su conjunto, válidos para postular universales separados (Ideas). Sin embargo, al mismo tiempo que esta crítica podría 
contribuir a mostrar la validez de los argumentos, también pone en riesgo su solidez. Es muy probable que el costo de aceptar Ideas de artefactos sea insostenible y que, por tanto, los argumentos no puedan considerarse como exitosos en última instancia.*

\section{BIBLIOGRAFÍA}

Allen, R. E., "Participation and Predication in Plato's Middle Dialogues", en R. E. Allen (ed.), Studies in Plato's Metaphysics, Londres, Routledge and Kegan Paul, 1965, pp. 43-60.

Caston, V., "Review of Gail Fine: On Ideas", Mind, vol. 104, 1995, pp. 162-166.

Cherniss, H., Aristotle's Criticism of Plato and the Academy, Baltimore, The Johns Hopkins Press, 1944.

CoOPer, N., "Between Knowledge and Ignorance", Phronesis, 31, 1986, pp. 229-242.

Devereux, D., "Separation and Immanence in Plato's Theory of Forms", Oxford Studies in Ancient Philosophy, 12, 1994, pp. 63-90.

Dooley, W. E., Alexander of Aphrodisias: On Aristotle, Metaphysics I, Londres, Duckworth, 1989.

FINE, G., "Separation", Oxford Studies in Ancient Philosophy, vol. 2, 1984, pp. 31-87.

-, "Immanence", Oxford Studies in Ancient Philosophy, vol. sup., 1986, pp. 71-97.

-, On Ideas: Aristotle's Criticism of Plato's Theory of Forms, Oxford, Clarendon Press, 1993.

Frank, D. H., The Arguments 'From the Sciences' in Aristotle's Peri Ideōn, Nueva York, Peter Lang, 1984.

\footnotetext{
* Quisiera agradecer por sus útiles comentarios a Axel Barceló, Enrique Hülsz, María Teresa Padilla y a José Antonio Robles. De manera muy especial le doy las gracias a Ricardo Salles por el apoyo que me ha brindado para la elaboración de este trabajo y por los valiosos comentarios que me ha proporcionado sobre el mismo. También deseo manifestar mi agradecimiento al Programa de Becas Posdoctorales de la Universidad Nacional Autónoma de México, a la Coordinación de Humanidades de dicha dependencia y al Instituto de Investigaciones Filosóficas de la UNAM por el apoyo recibido a través de una beca posdoctoral, la cual me ha permitido concluir la redacción de este artículo.
} 
HARLFINGER, D., "Edizione critica del testo del 'De Ideis' di Aristotele”, en W. Leszl, Il 'De Ideis' di Aristotele, e la teoria platonica delle idee, Florencia, Olschki, 1975, pp. 15-39.

HintikKa, J., "Plato on Knowing How, Knowing That and Knowing What", en J. Hintikka, Knowledge and the Known, Dordrecht, D. Reidel, 1974, pp. 31-49.

Isnardi Parente, M., "Le Peri Ideōn d'Aristote, Platon ou Xénocrate?", Phronesis, vol. 26, 1981, pp. 135-152.

Morrison, D., "Separation in Aristotle's Metaphysics", Oxford Studies in Ancient Philosophy, 3, 1985, pp. 125-157.

Robin, L., La Théorie platonicienne des idées et des nombres d'après Aristote, Paris, Félix Alcan, 1908.

Ross, D., Plato's Theory of Ideas, Oxford, Clarendon Press, 1951.

Santa Cruz, M. I., M. I. Crespo y S. Di Camillo, Las críticas de Aristóteles a Platón en el tratado Sobre las Ideas, Buenos Aires, Eudeba, 2000.

SAntas, G. X., "Hintikka on Knowledge and its Objects", en J. M. E. Moravcsik (ed.), Patterns in Plato's Thought, Dordrecht, D. Reidel, 1973, pp. 31-51.

Vlastos, G., “'Separation' in Plato”, Oxford Studies in Ancient Philosophy, 5, pp. 1987, pp. 187-196.

White, F. C., “The 'Many' in Republic 475a-480a", Canadian Journal of Philosophy, 7, 1977, pp. 291-306.

-, "The Scope of Knowledge in Republic V", Australasian Journal of Philosophy, 62, 1984, pp. 339-354. 